\title{
Metabolic engineering of 2-phenylethanol pathway producing fragrance chemical and reducing lignin in Arabidopsis
}

\author{
Guang $\mathrm{Qi}^{1} \cdot$ Dian Wang ${ }^{1,3} \cdot \mathrm{Li} \mathrm{Yu}{ }^{1} \cdot$ Xianfeng Tang $^{1,3} \cdot$ Guohua Chai $^{1}$. \\ Guo $\mathrm{He}^{1} \cdot$ Wenxuan $\mathrm{Ma}^{1} \cdot$ Shengying $\mathrm{Li}^{1} \cdot$ Yingzhen $\mathrm{Kong}^{2} \cdot$ Chunxiang $\mathrm{Fu}^{1}$ • \\ Gongke Zhou ${ }^{1}$
}

Received: 11 February 2015/Revised: 25 March 2015/Accepted: 31 March 2015/Published online: 21 April 2015

(c) Springer-Verlag Berlin Heidelberg 2015

\begin{abstract}
Key message Two 2-phenylethanol biosynthetic pathways were constructed into Arabidopsis; 2-phenylethanol biosynthesis led to reduced rate of lignin biosynthesis and increased cellulose-to-glucose conversion in the transgenic plants.

Abstract Lignin is the second most abundant biopolymer on the planet with importance for various agro-industrial activities. The presence of lignin in cell walls, however, impedes biofuel production from lignocellulosic biomass. The phenylpropanoid pathway is responsible for the biosynthesis of lignin and other phenolic metabolites such as 2-phenylethanol. As one of the most used fragrance chemicals, 2-phenylethanol is synthesized in plants from Lphenylalanine which is the first specific intermediate
\end{abstract}

Communicated by Q. Zhao.

G. Qi and D. Wang contributed equally to this work.

Chunxiang Fu

fucx@qibebt.ac.cn

$\triangle$ Gongke Zhou

zhougk@qibebt.ac.cn

Guang Qi

qiguang@qibebt.ac.cn

1 Key Laboratory of Biofuels and Shandong Provincial Key Laboratory of Energy Genetics, Qingdao Institute of Bioenergy and Bioprocess Technology, Chinese Academy of Sciences, Qingdao 266101, China

2 Key Laboratory of Tobacco Gene Resource, Tobacco Research Institute, Chinese Academy of Agricultural Sciences, Qingdao 266101, China

3 University of Chinese Academy of Sciences, Beijing 100049, China towards lignin biosynthesis. Thus, it is interesting to prove the concept that the phenylpropanoid pathway can be modulated for reduction of lignin as well as production of natural value-added compounds. Here we conferred two 2-phenylethanol biosynthetic pathways constructed from plants and Saccharomyces cerevisiae into Arabidopsis. As anticipated, 2-phenylethanol was accumulated in transgenic plants. Moreover, the transformants showed $12-14 \%$ reduction in lignin content and 9-13\% increase in cellulose content. Consequently, the glucose yield from cell wall hydrolysis was increased from $37.4 \%$ in wild type to $49.9-52.1 \%$ in transgenic plants with hot water pretreatment. The transgenic plants had normal development and even enhanced growth relative to the wild type. Our results indicate that the shunt of L-phenylalanine flux to the artificially constructed 2-phenylethanol biosynthetic pathway most likely reduced the rate of lignin biosynthesis in Arabidopsis.

Keywords Biofuel · Fragrance chemical · Lignin biosynthesis $\cdot$ Phenylpropanoid pathway $\cdot 2$-Phenylethanol

\section{Introduction}

Lignocellulosic biomass represents the most abundantly available renewable materials on Earth for the pulping and paper-making, ruminant animal feed and biofuel production ( $\mathrm{Li}$ et al. 2008). It consists of cell wall that makes up more than $80 \%$ of plant dry matter biomass (Ragauskas et al. 2014). Plant cell wall, mainly containing cellulose, hemicellulose and lignin, is highly recalcitrant to chemical or biological degradation due to its rigid and compact structure (Chen and Dixon 2007; Eudes et al. 2014). Lignin is a complex branched polymer of phenolic alcohols that 
plays an important role in cell wall structure reinforcement, mechanical support, water transport, and plant defense against biotic and abiotic stress (Campbell and Sederoff 1996; Douglas 1996; Boerjan et al. 2003). Lignin content and composition, however, have been recognized for its negative impact on a wide range of industrial applications, such as pulp, livestock forage, and bioethanol production (Chen and Dixon 2007; Li et al. 2010).

Currently, monolignol biosynthesis has been relatively well understood in plants. The monolignols are formed from L-phenylalanine via the phenylpropanoid pathway. Enzymes are known that can catalyze these reactions, involving phenylalanine ammonia lyase (PAL), hydroxylases, $O$-methyltransferases and reductase (Vanholme et al. 2010; Van Acker et al. 2013). Genetic manipulation of lignin is a promising strategy to reduce cell wall recalcitrance and therefore increase saccharification of lignocellulosic biomass (Bonawitz and Chapple 2013; Bonawitz et al. 2014). Previous studies have shown that downregulation of lignin biosynthetic genes in spruce, poplar, tobacco, alfalfa, switchgrass, and ryegrass can successfully reduce lignin biosynthesis and magnificently increase pulping efficiency, forage digestibility and bioethanol production (Sewalt et al. 1997a, b; Guo et al. 2001a, b; Reddy et al. 2005; Jouanin et al. 2000; Sarath et al. 2008; Fu et al. 2011a; Samuel et al. 2014; Louie et al. 2010; Tu et al. 2010). Other studies have indicated that overexpression of MYB transcription factor Atmyb4 or its homologs in Arabidopsis, tobacco and switchgrass leads to strong suppression of lignin biosynthesis (Shen et al. 2012). The above traditional strategies for lignin manipulation focus on the identification and regulation of lignin genes or their transcription factors. The available targets employed for lignin modification, however, are limited due to the number of genes known in monolignol biosynthetic pathways. Particularly, the genes that can cause a substantial decrease in lignin biosynthesis without major visible defects in plant growth are not sufficient for the purpose of commercial production of low lignin biomaterials (Bonawitz et al. 2014). Thus, the major challenge in current lignin bioengineering is identification of numerous novel targets or reconstruction of new pathways to partially alter the substantial carbon flux into lignin pathway (Li et al. 2008; Bonawitz and Chapple 2013). Previous studies have shown that disruption of $S$-adenosyl-L-methionine synthetases (SAMS) or methylenetetrahydrofolate reductase (MTHFR) in plants can affect the biosynthesis of SAM, the methyl donor consumed by two $O$-methyltransferases in lignin biosynthetic pathway, and therefore significantly reduce lignin content (Shen et al. 2002; Tang et al. 2014). Another promising structure-based protein engineering approach indicates that expression of an engineered monolignol 4- $\mathrm{O}$ methyltransferase created by iterative saturation mutagenesis in Arabidopsis can result in etherealization of the para-hydroxyls of lignin monomeric precursors, and therefore lead to depression of lignin biosynthesis and improvement of cell wall saccharification (Zhang et al. 2012). Other studies have suggested that lignin polymerization can be reduced through the overproduction of sidechain-truncated lignin monomers achieved by expressing a bacterial hydroxycinnamoyl-CoA hydratase-lyase (HCHL) in lignifying tissues of Arabidopsis inflorescence stems and improve saccharification (Eudes et al. 2012).

2-PE is one of the most used flavor principles with a pleasant rose-like odor. Several plants such as rose, carnation, hyacinth, and jasmine are capable of producing natural 2-PE. However, those plant tissues usually contain trace amounts of 2-PE, except rose flower (Rusanov et al. 2005). Thus, the majority source of 2-PE currently in use is synthesized by chemical means. Although there is no difference between the synthetic 2-PE and the natural one, the increasing demand for natural flavors makes biotechnological production of 2-PE an interesting option. 2-PE is a general metabolite of microbial fermentation. Previous studies have shown that microorganisms can convert Lphenylalanine (L-phe) to 2-PE in their culture via the Ehrlich pathway (Hazelwood et al. 2008). Three enzymes, transaminase, decarboxylase, and dehydrogenase, are known in this route which is by transamination of L-phe to phenylpyruvate, followed by decarboxylation to phenylacetaldehyde and reduction to 2-PE. 2-PE biosynthetic pathway in plants, by contrast, is yet to be clearly elucidated. Three plausible pathways are proposed for 2-PE biosynthesis in a variety of different plant species (Tieman et al. 2007). The first pathway consists of an enzyme of CYP79 family responsible for the oxidative decarboxylation of L-phe to produce phenylacetaldoxime. Phenylacetaldoxime is successively hydrolyzed to yield phenylacetaldehyde (PAld) which is reduced to 2-PE by an alcohol dehydrogenase (ADH) or PAld reductase (PAR). PAR has been identified in tomato and is designated as LePAR1 (Tieman et al. 2007). The second pathway was first found in tomato fruits, where an aromatic L-amino acid decarboxylase (AADC) converts L-phe to 2-phenylethylamine $\left(2 \mathrm{PNH}_{2}\right)$. A monoamine oxidase $(\mathrm{MAO})$ further catalyzes the conversion of $2 \mathrm{PNH}_{2}$ to pAld which is then transformed to 2-PE by PAR. The third pathway involves a direct conversion of L-phe to PAld by a bifunctional phenylacetaldehyde synthase (PAAS) which is a petunia AADC reported by Kaminaga et al. (2006), and 2-PE is formed from PAld by PAR.

The aim of our present work was to reduce carbon flux to lignin biosynthesis by introducing 2-PE biosynthetic pathway into Arabidopsis. The enzymes in novel pathways were recruited from Saccharomyces cerevisiae, tomato, and petunia. Among them, ARO9 (transaminase, 
Hazelwood et al. 2008) and PAAS will, respectively, compete with PAL for L-phe, the initial monolignol precursor. We expect that the altered carbon flux towards lignin pathway would reduce lignin biosynthesis. Thus, lignin content, 2-PE and its derivatives were determined in transgenic Arabidopsis plants. Furthermore, we detected cellulose content, matrix polysaccharide composition, and cell wall saccharification efficiency to study the impact of the reconstructed 2-PE pathway on other cell wall components and biomass recalcitrance of transgenic Arabidopsis plants.

\section{Materials and methods}

\section{Plant materials and growth conditions}

Arabidopsis thaliana ecotype Columbia (Col-0) was used in this study. Arabidopsis plants were grown in greenhouse under $16 \mathrm{~h} \mathrm{light} / 8 \mathrm{~h}$ dark at $22{ }^{\circ} \mathrm{C}$ with $65 \%$ relative humidity. Seeds were sterilized before sown on half-strength MS medium. After stratification at $4{ }^{\circ} \mathrm{C}$ for 2 days, Arabidopsis seeds were germinated at $22{ }^{\circ} \mathrm{C}$. Tomato ( $S$. lycopersicum $\mathrm{cv}$. M82) and petunia ( $P$. hybrida, $\mathrm{cv}$. Mitchell Diploid) plants were grown in greenhouse with day/night temperatures of $22 / 17{ }^{\circ} \mathrm{C}$ under $16 \mathrm{~h}$ light $/ 8 \mathrm{~h}$ dark. Tomato mature fruits and petunia flowers were harvested and immediately frozen in liquid nitrogen and stored at $-80{ }^{\circ} \mathrm{C}$.

\section{Chemicals and reagents}

Taq DNA polymerase and all restriction enzymes were purchased from MDbio (Taiwan) and New England Biolabs (USA). TRIzol reagent for RNA isolation was from Invitrogen (USA). RNase-free DNase and the kits used for cDNA synthesis and RT-PCR were from Thermo Fisher (USA) and TransGen (Beijing, China). The kits used for molecular cloning were from Takara (Japan) or Thermo Fisher (USA). Oligo nucleotides synthesis and DNA sequencing were performed by Sunnybio (Shanghai, China). The other chemicals used for molecular biology and phytochemistry analysis were purchased from Sigma-Aldrich (USA).

\section{Strains and plasmids}

The Gateway entry vector, pEN-L4-2-L3, and the plant destination vectors, pK7m34GW2-8m21GW3 and pK7m34GW2-8m21GW3-9m56GW4 were purchased from VIB/Gent (Belgium). The Gateway entry vector pDONR P5-P6 was purchased from Invitrogen (USA). Agrobacterium strain GV3101 was used for plant transformation. S. cerevisiae strain was used for gene cloning.

\section{Gene cloning and vector construction}

The ARO9, ARO10, and $A D H 2$ genes were amplified from the genomic DNA of $S$. cerevisiae using gene-specific primers as follows: ARO9 (forward, 5'-ATGACTGC TGGTTCTGCCCCC- $3^{\prime}$; reverse, 5'-TCAACTTTTATAG TTGTCAAAAAAT- $3^{\prime}$ ), ARO10 (forward, 5'-ATGGCA CCTGTTACAATTGAAAAG- $3^{\prime}$; reverse, 5'-CTATTTT TTATTTCTTTTAAGTGC- $3^{\prime}$ ), and $A D H 2$ (forward, $5^{\prime}$-A TGTCTATTCCAGAAACTCAAAAAG- $3^{\prime}$; reverse, $5^{\prime}$-TT ATTTAGAAGTGTCAACAACGTATC- $3^{\prime}$ ). The amplified fragments were ligated to the Gateway entry vector pDONR P5-P6, pEN-L4-2-L3, and pGWC-T, sequenced, and then transferred into the Gateway binary vector pK7m34GW2-8m21GW3-9m56GW4 using the Gateway recombination system (Invitrogen) (Karimi et al. 2007). The PAAS and LePARl genes were isolated from the cDNAs of petals of $P$. hybrida (cv. Mitchell) and mature fruits of $S$. lycopersicum using the gene-specific primers as follows: PAAS (forward, 5'-ATGGATACTATCA AAATCAACCCAG- $3^{\prime}$; reverse, 5'-CTACGCATTCAGC ATCATAGTTG-3') and LePARl (forward, 5'-ATG AGTGTGACAGCGAAAACAGTG- $3^{\prime}$; reverse, 5'-TTAC ATAGAAGATGAACCTCCAAA- $3^{\prime}$ ). The amplified fragments of PAAS and LePAR1 were ligated to pEN-L4-2-L3, and pGWC-T, respectively, and transferred into the Gateway binary vector pK7m34GW2-8m21GW3.

\section{RNA isolation and RT-PCR}

Total RNA extraction and RT-PCR was conducted as described previously (Qi et al. 2013). Briefly, 7-week-old Arabidopsis stems were collected and extracted with TRIzol reagent (Invitrogen) according to manufacturer's instructions. RNA was digested with DNase I (Sigma) to remove genomic DNA contamination, and the first-strand cDNA was reverse-transcribed with total RNA $(2 \mu \mathrm{g})$ using RevertAid First-Strand cDNA Synthesis Kit (Thermo Fisher) and oligo-dT primers. Beacon Designer v7.0 (Premier Biosoft International) was used to design the genespecific primers as follows: ARO9 (forward, 5'-TGC CCGTGTCATCCGTTTGG-3'; reverse, 5'-AAGTTGGACTCAGCCATTGCCTTT-3'), ARO10 (forward, 5'-C CCTGGTGATGTTGTCGTTTGTGAAA- ${ }^{\prime}$; reverse, $5^{\prime}$-A TTGATGTGAGCGTTTGAGTGGTCTTG- $\left.3^{\prime}\right), \quad A D H 2$ (forward, 5'-GTTCAAGCCGCTCACATTCCTCAA- $3^{\prime}$; reverse, 5'-TAGACCACCAGCAGCACCAGAA- $\left.3^{\prime}\right), P A A S$ (forward, $5^{\prime}$-CTCAGAAATTTCATAAGAAGC- ${ }^{\prime}$; reverse, 5'-ATCATAGTTGCATGGTTTCGAA-3') and LePARI (forward, 5'-TCCTCTTTTGGGTGGGTTAACGT-3'; 
reverse, 5'-CTCCTTTGATACTTGATAATTTTG-3'). The expression of the AtACTIN2 gene was used as an internal control.

\section{Histochemistry assay}

7-week-old Arabidopsis basal stems were cut and fixed with $4 \%$ paraformaldehyde at $4{ }^{\circ} \mathrm{C}$ overnight. After fixation, the tissues were dehydrated in a graded ethanol series, and embedded in paraplast as described previously (Chai et al. 2014). The paraplast-embedded stems were sectioned to a thickness of $10 \mu \mathrm{m}$ using a Leica RM 2235 microtome (Leica). The dewaxed and rehydrated sections were incubated for $5 \mathrm{~min}$ in the solution of Phloroglucinol (Sigma) in $20 \% \mathrm{HCl}$ and rinsed with water (Pomar et al. 2002). All sections were observed at bright field with an Olympus BX-51 microscope equipped with an OLYMPUS DP26 digital camera and OLYMPUS DP2-BSW software.

\section{Cell wall residues preparation}

The inflorescence stems of 10-week-old mature senesced tissues were collected $3 \mathrm{~cm}$ above the base for cell wall residues (CWRs). The senesced stems were harvested and lyophilized, and the dried materials were then grinded in ball mill (Retsch). The ground-well stem materials were thoroughly washed with chloroform:methanol (2:1), $100 \%$ methanol, $50 \%$ methanol, and MiliQ water, and then dried in vacuum machine (Fu et al. 2011b). De-starching was performed by treating CWR with pullulanase M3 (0.5 U $\mathrm{mg}^{-1}$, Megazyme) and $\alpha$-amylase $\left(0.75 \mathrm{U} \mathrm{mg}^{-1}\right.$, Sigma $)$ in 0.1 M NaOAc buffer (pH 5.0) overnight (Li et al. 2009).

\section{Lignin analysis}

Total lignin content was determined by the $\mathrm{AcBr}$ method (Foster et al. 2010). Briefly, dried-well CWR samples were reacted with freshly prepared acetyl bromide reagent at $50{ }^{\circ} \mathrm{C}$ for $4 \mathrm{~h}$. After centrifugation at $3500 \mathrm{~g}$ for $15 \mathrm{~min}$, the upper layer was quantitatively transferred and reacted with $2 \mathrm{~mol} / \mathrm{L} \mathrm{NaOH}$ and $0.5 \mathrm{~mol} / \mathrm{L}$ hydroxylamine. The samples were diluted with acetic acid, and the absorptions at $280 \mathrm{~nm}$ were determined with a NanoDrop ${ }^{\circledR}$ ND-1000 spectrophotometer (Thermo Scientific). AcBr lignin content was calculated by means of the Bouguer-LambertBeer law in five biological duplicates.

\section{Cellulose content assay}

Cellulose content was determined using the method as described previously (Foster et al. 2010). Briefly, CWR was hydrolyzed by trifluoroacetic acid (TFA) at $120{ }^{\circ} \mathrm{C}$ for $120 \mathrm{~min}$. The TFA resistant materials were treated with
Updegraff reagent (acetic acid: nitric acid: water, 8:1:2, $\mathrm{v} / \mathrm{v}$ ) at $100{ }^{\circ} \mathrm{C}$ for $30 \mathrm{~min}$, and the resulting pellets were completely hydrolyzed using $67 \% \mathrm{H}_{2} \mathrm{SO}_{4}(\mathrm{v} / \mathrm{v})$. The released glucose was measured using a glucose assay kit (Cayman Chemical, MI) with a dehydration factor of 0.9.

\section{Matrix polysaccharide composition analysis}

Matrix polysaccharide composition analysis was performed with TFA-hydrolyzed materials as described previously (Yu et al. 2010). The released monosaccharides were derived by 1-phenyl-3-methyl-5-pyrazolone (PMP), and the derivatives were analyzed by high-performance liquid chromatography (HPLC).

\section{Cell wall pretreatment and saccharification}

Pretreatments and saccharification of CWR of 10-week-old senesced Arabidopsis stems were performed as described previously with minor modifications (Van Acker et al. 2013). Ball-milled CWR of senesced stems (50 mg) was incubated in glass culture tubes containing $2 \mathrm{~mL}$ water at $30{ }^{\circ} \mathrm{C}$ for $30 \mathrm{~min}$ and autoclaved at $120{ }^{\circ} \mathrm{C}$ for $1 \mathrm{~h}$. Saccharification was initiated by the addition of $1.5 \mathrm{~mL}$ of $100 \mathrm{mM}$ citrate buffer at $\mathrm{pH} 4.8,0.5 \%$ w/w cellulase complex NS50013 and $0.5 \%$ w/w glucosidase NS50010 (Novozymes, Bagsværd, Denmark). After $24 \mathrm{~h}$ of incubation at $50{ }^{\circ} \mathrm{C}$ with $100 \mathrm{rpm}$ shaking, the samples were centrifuged at $15,000 \mathrm{~g}$ for $10 \mathrm{~min}$, and $100 \mu \mathrm{L}$ of the supernatant was collected for glucose measurement using a glucose assay kit (Cayman Chemical, MI).

\section{Quantification of 2-phenylethanol in the transgenic Arabidopsis plants}

Rosette leaves and 7-week-old stems were collected and frozen individually in liquid nitrogen, and then grinded in ball mill (Retsch) and kept at $-80^{\circ} \mathrm{C}$. For the 2-PE analyses, samples were extracted using methyl-tert-butyl ether with $0.5 \mathrm{mM}$ benzyl methyl ether as internal standard, and the extracts were individually analyzed with an HP 6890 Series GC System equipped with a RESTEK-5Sil-MS column using the method as described previously (Tieman et al. 2007). 2-PE was quantified based on $\mathrm{m} / \mathrm{z}, 122$ and 91 extracted ion traces and areas normalized to benzyl methyl ether peak area and quantified using external calibration with authentic 2-PE standard. Each chemical analysis data point is the average of five independent transgenic lines.

\section{Phenolics profiling analysis}

Phenolics profiling analysis was determined using the method adapted from Fu et al. (2011b). Briefly, samples 
were extracted with methanol:water $(8: 2, \mathrm{v} / \mathrm{v})$ containing $0.5 \mathrm{mM}$ naringenin as internal standard and analyzed using liquid chromatography electrospray ionization mass spectrometry (LC-ESI-MS/MS). An Agilent 1290 Infinity LC coupled to a Bruker Esquire Ion-trap Mass Spectrometer equipped with an electrospray ionization source (ESI) system (Agilent Technologies, Palo Alto, CA) was employed. Mass determination was conducted by ESI in negative ion polarity. Mass spectra were recorded over the range $50-2200 \mathrm{~m} / \mathrm{z}$.

\section{Statistical analysis}

Triplicate samples were collected for each transgenic line. The mean values were used for statistical analyses. Data from each trait were subjected to one-way ANOVA. The significance of treatments was tested at the $P<0.05$ level.

\section{Results}

\section{Introduction of 2-PE biosynthetic pathway into Arabidopsis plants}

2-PE biosynthesis from L-phenylalanine is involved in different pathways in plants and $S$. cerevisiae. To assess the relative efficiency of each pathway for 2-PE production in
Arabidopsis, both pathways were reconstructed and introduced into Arabidopsis, respectively. In $S$. cerevisiae, the Ehrlich pathway for 2-PE biosynthesis consists of transaminase, 2-keto-acid decarboxylase, and alcohol dehydrogenase (Fig. 1a). Three corresponding genes (ARO9, $A R O 10$, and $A D H 2$ ) that encoded these enzymes were selected and amplified from the genomic DNA of $S$. cerevisiae, and constructed in the binary vector pK7m34GW28m21GW3-9m56GW4 (Karimi et al. 2007; Hazelwood et al. 2008), which contains three cassettes with different promoters and terminators that work well in plants (Fig. 1b). The 2-PE biosynthetic pathway in plants requires at least two genes (PAAS and PAR) (Fig. 1a). Accordingly, the $P A A S$ gene was isolated from $P$. hybrida (cv. Mitchell) petals, and $P A R$ was amplified from $S$. lycopersicum (LePAR1, Sakai et al. 2007) mature fruits. The two genes were constructed into the binary vector pK7m34GW28m21GW3, which has two cassettes with different promoters and terminators (Fig. 1b). Arabidopsis plants were transformed with the two vectors, respectively. Independent T1 kanamycin-resistant plants were screened for the insertion of genes of the two pathways using genomic PCR. RT-PCR analysis further revealed high expression levels of the recruited genes in transgenic Arabidopsis plants (Fig. 2b). Five homozygous lines containing ARO9/ ARO10/ADH2 or PAAS/LePAR1 were separately selected for further analysis.

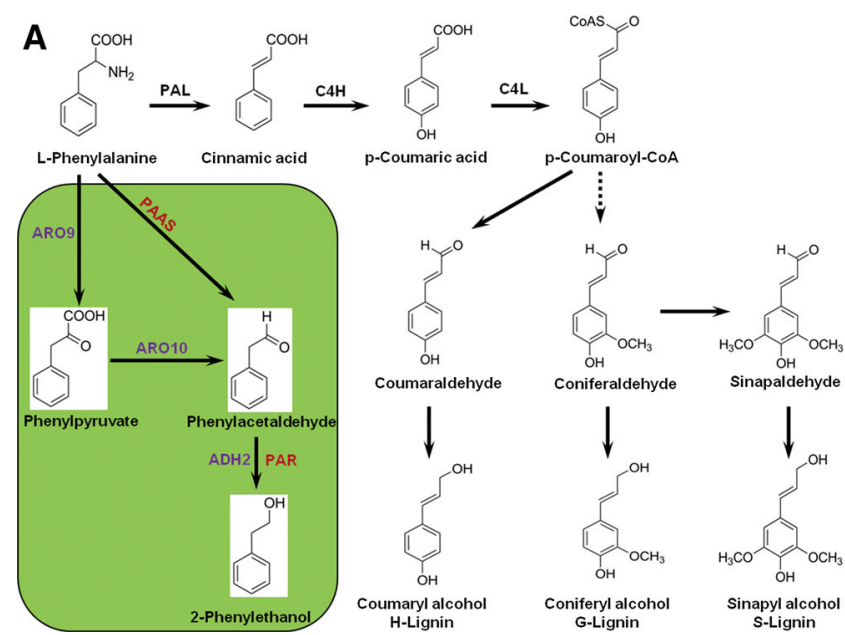

B

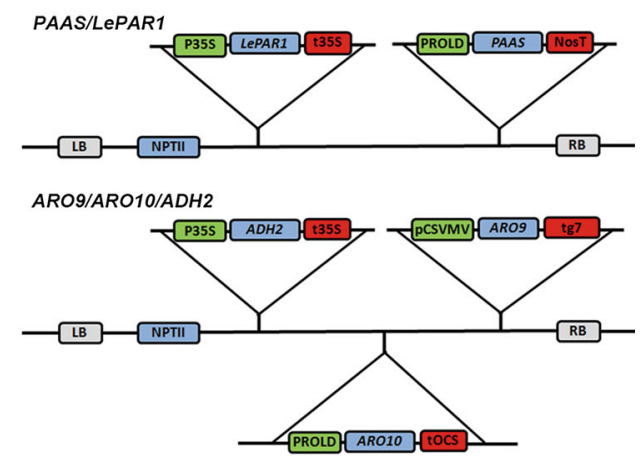

Fig. 1 Recruitment of enzymes for construction of 2-phenylethanol biosynthetic pathways in Arabidopsis. a Proposed scheme of routes for 2-phenylethanol biosynthesis. The enzymes ARO9, ARO10, and $\mathrm{ADH} 2$ highlighted in purple were recruited from Saccharomyces cerevisiae. The enzymes PAAS and PAR highlighted in red were from $P$. hybrida and $S$. lycopersicum, respectively. ARO9, aromatic aminotransferase II; ARO10, 2-keto-acid decarboxylase; ADH2, alcohol dehydrogenase-2; PAAS, phenylacetaldehyde synthase; PAR, phenylacetaldehyde reductase. Phenylalanine ammonia lyase (PAL), cinnamate 4-hydroxylase $(\mathrm{C} 4 \mathrm{H})$ and 4-coumarate coenzyme A:ligase (4CL) are the enzymes directing carbon flux towards lignin biosynthesis. b Design of the binary vector constructs containing

2-phenylethanol biosynthetic genes isolated from Saccharomyces cerevisiae and plants. Two proposed 2-phenylethanol biosynthetic pathways were recruited, and the genes were inserted into the Gateway destination vector pK7m34GW2-8m21GW3 and pK7m34GW2-8m21GW3-9m56GW4, respectively. LB, the left border of T-DNA; RB, the right border of T-DNA; NPT II, the kanamycin resistance gene; P35S and t35S, Cauliflower Mosaic Virus promoter and terminator sequences; PROLD, Agrobacterium rhizogenes promoter sequence; tOCS, Agrobacterium tumefaciens octopine synthase terminator sequence; pCSVMV, Cassava vein mosaic virus promoter sequence; tg7, Agrobacterium tumefaciens g7 terminator sequence 

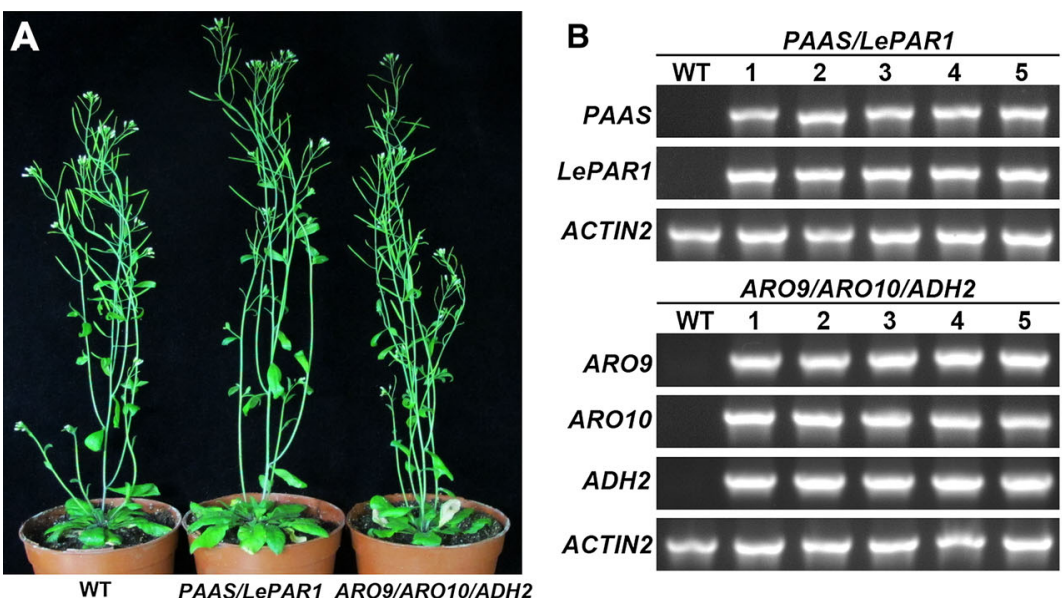

Fig. 2 Phenotypes of the transgenic plants. a 7-week-old PAAS/ LePAR1, ARO9/ARO10/ADH2, and wild-type (WT) plants. b Expression analysis of the 2-PE biosynthetic genes in 7-week-old transgenic plants. RT-PCR of the target genes in the PAAS/LePAR1 and ARO9/

The growth of transgenic lines resembles the wild-type plants. Both ARO9/ARO10/ADH2 and PAAS/LePAR1 lines had similar plant height and biomass yields (Fig. 2a, c).

\section{Reduction of lignin content in transgenic Arabidopsis plants}

The recruited ARO9 and PAAS in 2-PE biosynthetic pathway consume L-phe, the monolignol precursor, and compete with PAL for the carbon flux towards lignin pathway in transgenic Arabidopsis plants. To study the impact of 2-PE pathway on lignin biosynthesis, we first stained the transverse cross-sections of stem tissues of transgenic and wild-type plants with phloroglucinol- $\mathrm{HCl}$ solution. Histochemistry assay revealed low lignin deposition in sclerenchyma tissues of transgenic plants compared with wild type (Fig. 3a). Furthermore, the $\mathrm{AcBr}$ lignin contents of transgenic and the wild-type plants were determined by wet-chemistry analysis. Compared with wild-type plants, transgenic plants showed $12-14 \%$ reduction in lignin content (Fig. 3b).

\section{Effects of 2-PE biosynthesis on other cell wall components in transgenic Arabidopsis plants}

To study the effects of altered carbon flux resulted from 2-PE pathway on other cell wall components, we examined the content of cellulose and matrix polysaccharides in the transgenic and wild-type plants. We found that the transgenic plants contained 9-13\% more cellulose than the wild type (Fig. 4). Subsequently, we measured the matrix polysaccharide content in Arabidopsis stem CWR. The CWRs were hydrolyzed with trifluoroacetic acid (TFA) to

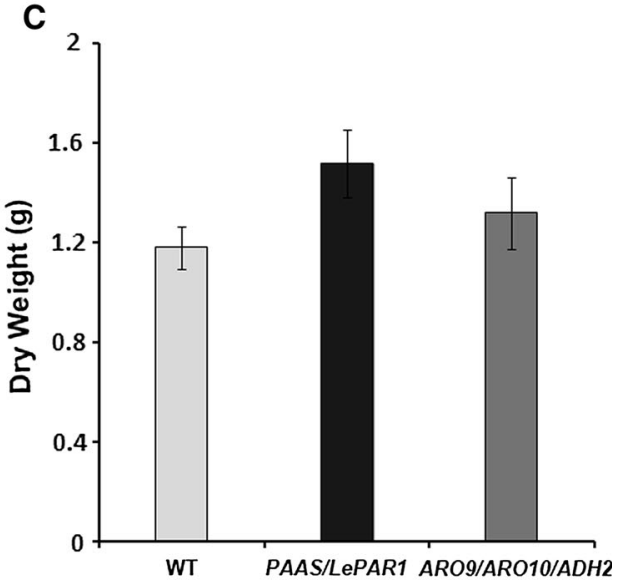

ARO10/ADH2 plants. ACTIN2 was used as an internal control. c Dry weights of the 10-week-old PAAS/LePAR1, ARO9/ARO1O/ADH2, and wild-type plants. Data are the mean $\pm \mathrm{SE}$ of 25 independent biological repeats

release monosaccharides from matrix polysaccharides and amorphous cellulose (Van Acker et al. 2013). No different matrix polysaccharide content was observed between transgenic and wild-type plants. Furthermore, we detected cell wall glycosyl residue composition in Arabidopsis stem CWR to study the effect of 2-PE biosynthesis on the matrix cell wall sugars. Although no difference in the total amount of monosaccharides released from CWR was examined between the transgenic and wild-type plants, the significant increases in mannose, rhamnose, and arabinose were found in transgenic plants, as well as a decrease in xylose (Table 1).

\section{Improved saccharification efficiency in transgenic Arabidopsis plants}

The reduced lignin content and increased cellulose amount suggest that the cell wall of transgenic materials were to be efficiently deconstructed to release more fermentable sugars. To evaluate saccharification efficiency of cell walls, the CWR of transgenic and wild-type plants was subjected to enzymatic hydrolysis with or without hot water pretreatment. Without pretreatment, about 31.0 and $30.1 \%$ glucose yield of total glucan were obtained for ARO9/ ARO1O/ADH2 and PAAS/LePARI transgenic materials in $24 \mathrm{~h}$, respectively, while wild-type materials have only $24.2 \%$ glucose yield, indicating that the changes of lignin biosynthesis in transgenic plants have a significant effect on saccharification of the unpretreated cell wall materials (Fig. 5a). With pretreatment, the transgenic materials released more amount of glucose (approximate 49.9 and $52.1 \%$ of total glucan, respectively) than that of wild-type materials (37.4\% of total glucan) (Fig. 5b). Moreover, the 
A

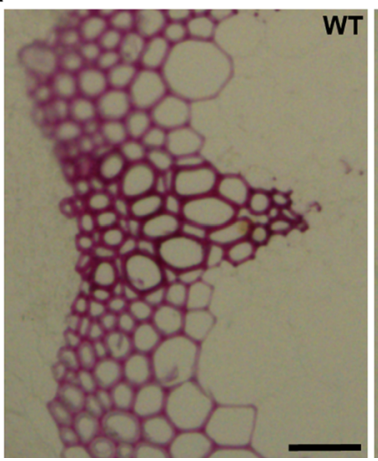

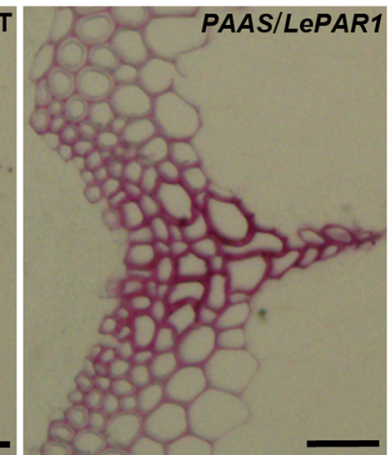

Fig. 3 Lignin content of stem cell wall materials from the transgenic and wild-type plants. a Histochemical staining using phloroglucinol$\mathrm{HCl}$ of stem sections from 7-week-old PAAS/LePAR1, ARO9/ARO10/ $A D H 2$, and wild-type plants. Bars $50 \mu \mathrm{m}$. b Lignin content in cell wall of 10-week-old stems from the transgenic and wild-type plants.

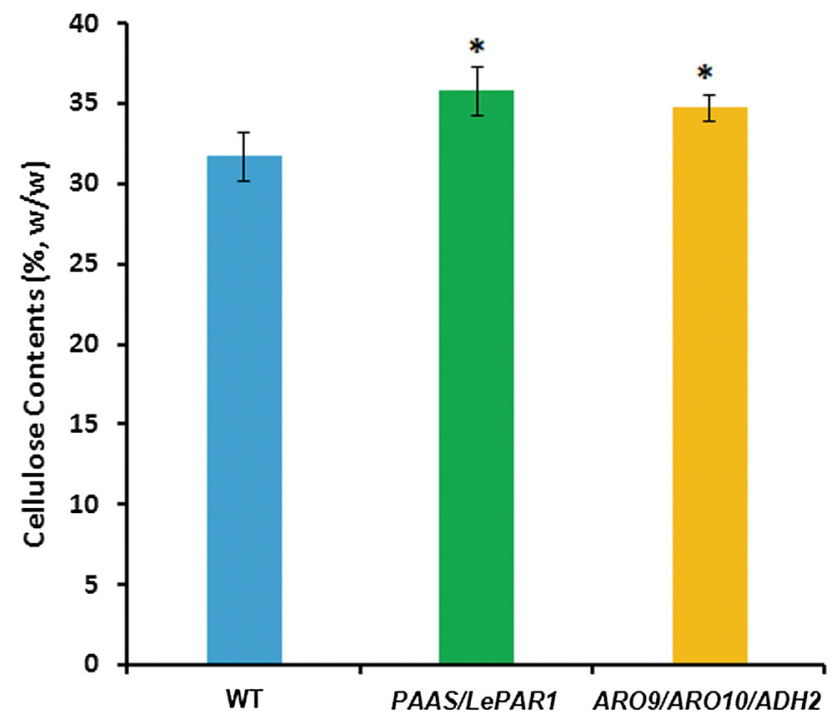

Fig. 4 Cellulose content of stem cell wall materials from the transgenic and wild-type plants. The cellulose contents of 10-weekold stem cell wall materials from PAAS/LePAR1, ARO9/ARO10/ $A D H 2$, and wild-type plants were determined by the Updegraff method (see Experimental procedures). The results presented are percentage CWR. Each data represents the mean \pm SE of five biological replicates. $* P<0.05$

transgenic materials had a percentage increase in cellulose conversion due to the pretreatment $(65.8$ and $68.1 \%$, respectively) higher than that of the wild type (54.5\%).

\section{Accumulation of 2-PE and lignin intermediates in transgenic Arabidopsis plants}

To examine the accumulation of 2-PE and its derivatives in transgenic Arabidopsis plants, rosette leaves and 7-weekold stems were collected separately for GC-MS and LCMS analyses. The transgenic plants were capable of

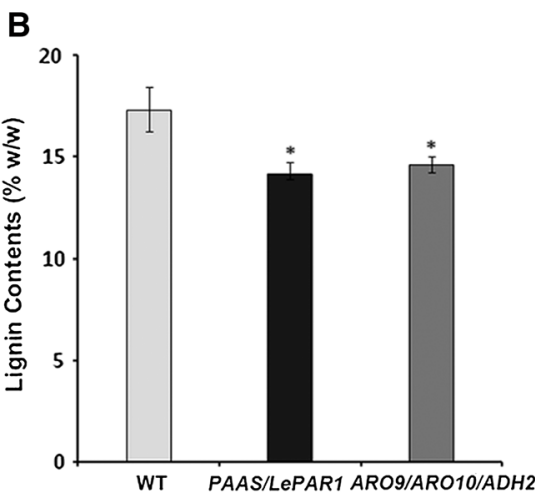

Lignin content was determined with CWR prepared from 10-weekold stems using the $\mathrm{AcBr}$ assay and expressed as the weight percentage $(w / w)$. Data are the mean $\pm \mathrm{SE}$ of five independent biological repeats. $* P<0.05$

producing 2-PE in rosette leaves and stems. The amount of 2-PE ranged from 0.0034 to $0.037 \%$ fresh weight in the PAAS/LePARI and ARO9/AROIO/ADH2 transgenic plants (Fig. 6). Moreover, the rosette leaves accumulated higher 2-PE than stems in transgenic plants. However, 2-PE was not found in wild-type plants (Fig. 6). Although the concentration of 2-PE was low in transgenic Arabidopsis tissues, our result revealed that 2-PE biosynthetic pathways reconstructed from plants and S. cerevisiae can work well in Arabidopsis plants. In addition, we determined lignin intermediates levels in transgenic and wild-type plants using LC-MS/MS. The transgenic plants accumulated normal amount of sinapoyl malate, syringin and coniferin, the major derivatives from the intermediates of lignin biosynthetic pathway (Data not shown).

\section{Discussion}

The derivatives from phenylpropanoid biosynthetic pathway are sustainable and renewable sources of biofuels, commodity chemicals, and pharmaceuticals. Monolignols are formed from L-phe and consume the majority of carbon flux through the phenylpropanoid pathway. Besides lignin, L-phe is the common precursor required for the biosynthesis of other phenolic compounds, such as 2-PE, vanillin, flavonoid, tannin, salicylic acid, and suberin (Vogt 2010). In our study, we recruited ARO9 and PAAS which can efficiently convert L-phe to phenylpyruvate and PAld, respectively, and reconstructed two 2-PE biosynthesis pathways including the enzymes isolated from $S$. cerevisiae, tomato, and petunia, respectively (Fig. 1). The transgenic Arabidopsis plants accumulated 2-PE metabolite substantially and showed reduced lignin content, increased cellulose amount, and improved saccharification efficiency. 


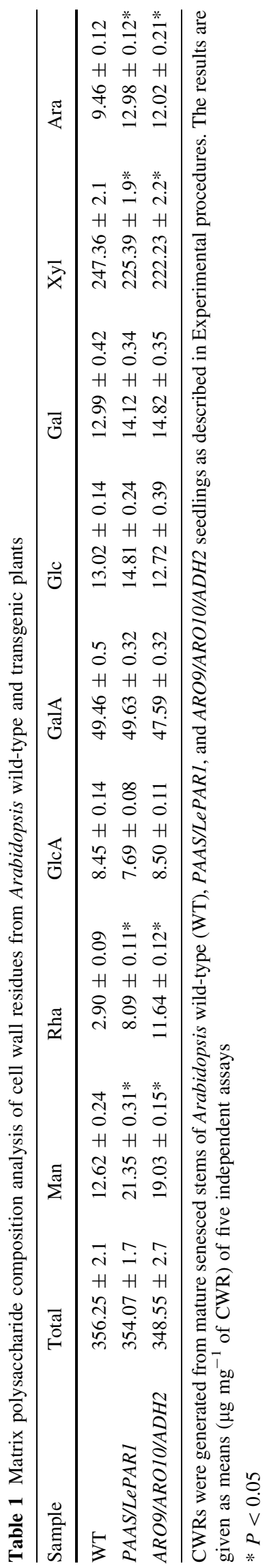

Numerous previously reported studies in lignin bioengineering targeted enzymes or transcription factors directly involved in lignin biosynthetic pathway. Genetic modification of lignin biosynthesis, particularly monolignol pathway, has been carried out during past decades ( $\mathrm{Li}$ et al. 2008). These data indicated that the severe reduction in lignin content was always accompanied with structural or developmental defects in plants such as thin cell wall, irregular xylem, dwarf, and lodging (Bonawitz et al. 2014). It has been suggested that an abnormal accumulation of lignin intermediates in lignin-deficient mutants may induce an inappropriate or exaggerated response via a homeostatic pathway and therefore repress cell wall lignification and impair plant growth (Bonawitz et al. 2014). Moreover, some lignin intermediates such as ferulic acid, vanillin, p-hydroxybenzaldehyde, and hydroxycinnamaldehyde can be integrated to the growing lignin polymer and affect plant phenotype under biotic or abiotic stress (Zhao et al. 2013). For example, the cell wall of Medicago CADI mutant is composed with substantial unconventional lignin derived from coniferaldehyde and sinapaldehyde. The mutants appear normal in the greenhouse under the standard temperature. However, the plants are dwarfed when grown at high temperature (Zhao et al. 2013). In our work, we did not find any abnormal accumulation of soluble phenolics derived from lignin intermediates in transgenic Arabidopsis plants. The biosynthesis of sinapoyl malate, syringin and coniferin utilizes the same intermediates as lignin biosynthetic pathway in Arabidopsis. Metabolic engineering of 2-phenylethanol in Arabidopsis reduced L-phe flux to monolignol biosynthetic pathway, and therefore resulted in the significant decrease in lignin content. Given the fact that lignin is the second most abundant polymer in the plant cell walls, whereas a low amount of sinapoyl malate, syringin and coniferin accumulates in Arabidopsis, it is reasonable that the remained lignin intermediates were sufficient for the normal biosynthesis of the above soluble phenolics, but not for lignin in the transgenic plants. Although the lignin content was significantly reduced in transgenic plants, their growth and development are normal under the standard conditions. The increased cellulose amount could compensate the biomass reduction caused by lignin reduction in cell wall complex. This hypothesis is supported by the previous study in poplar, which suggested that the deposition of lignin and cellulose could be coordinately regulated by a compensatory mechanism ( $\mathrm{Hu}$ et al. 1999). Notably, although the matrix polysaccharide content of transgenic Arabidopsis plants was virtually indistinguishable from that of wild-type plants, the decreased amount of xylose in transgenic plants, as well as the increased levels of mannose, rhamnose, and arabinose indicated that the composition of matrix polysaccharides was significantly altered in 


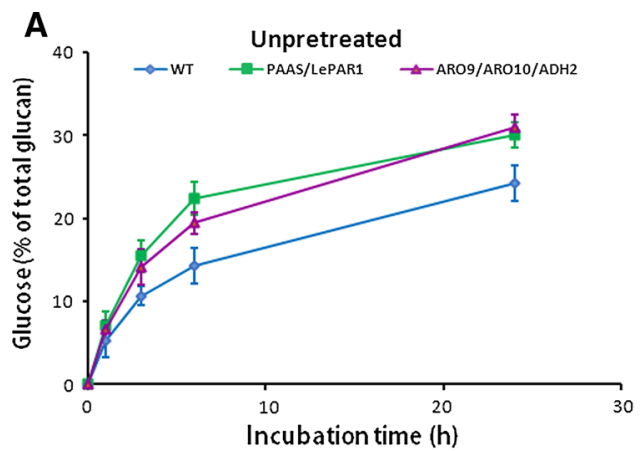

Fig. 5 Saccharification of stem cell wall materials from transgenic and wild-type plants with or without pretreatment. Enzymatic hydrolysis time-course of untreated (a) and hot water-pretreated (b) in 10-week-old stem cell wall materials of wild-type and

our transgenic materials (Table 1). This result is in accordance with previous observations in Arabidopsis CCRI and $\mathrm{C} 4 \mathrm{H}$ mutants, where disruption of lignin biosynthesis affected the yield of xylose, arabinose, and galactose derived from cell wall polysaccharides (Van Acker et al. 2013). The mechanism underlying the altered deposition of other cell wall polymers in lignin-deficient plants, however, is at present poorly understood but deserves further investigation.

Lignin has been identified as a critical factor negatively impacting on cell wall digestibility and saccharification (Chen and Dixon 2007; Eudes et al. 2014). Our results showed that lignin reduction resulted from the introduction of the 2-PE pathway in Arabidopsis plants facilitated breakdown of cell wall polysaccharides. The amount of glucose enzymatically released from unpretreated transgenic Arabidopsis materials was higher than that of wild type (Fig. 5a, 31.0 and $30.1 \%$, respectively). Furthermore, the cell wall materials were subjected to pretreatment with hot water to completely hydrolyze hemicellulose and slightly break non-condensed lignin. Both transgenic and wild-type Arabidopsis materials released more glucose with pretreatment (Fig. 5b). However, the pretreatment effect of transgenic materials was higher than that of wildtype materials, indicating that our transgenic materials had less recalcitrance to enzymatic hydrolysis and were more susceptible to the hot water pretreatment. In addition, no abnormal lignin intermediates are observed in our transgenic Arabidopsis plants, which would further facilitate high ethanol yield during downstream processing. It has been suggested that many residual soluble lignin pathwayderived constituents can lead to sensitivity and/or inhibition in the fermentation broth (Ximenes et al. 2010). Therefore, the ethanol production of our transgenic materials deserves additional evaluation in the future.

Although at least three plausible routes for 2-PE biosynthesis were proposed in plants (Tieman et al.

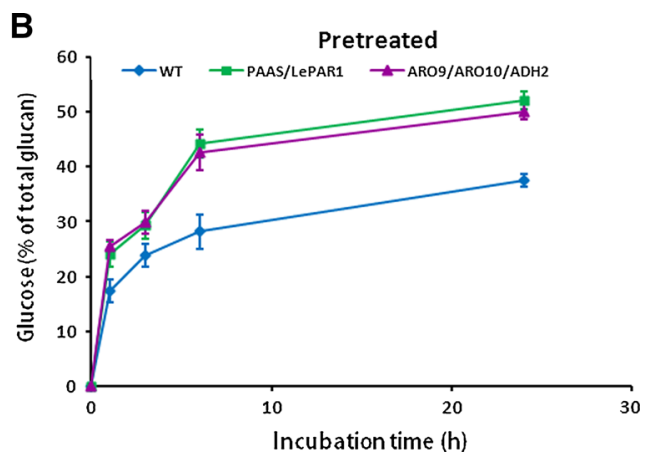

transgenic plants. Amounts of glucose released from biomass after a $24 \mathrm{~h}$ enzymatic digestion are shown. Values are mean $\pm \mathrm{SE}$ of five independent biological repeats. $* P<0.05$

2007), some key enzymes responsible for the postulated steps in the pathway to 2-PE have yet to be characterized. It have been confirmed that 2-PE is formed from Lphe in plants. Two gene families, CYP79 and AADC, encode enzymes converting L-phe to corresponding intermediates (Tieman et al. 2007). Because plants contain many CYP79 and AADC members, the certain members responsible for 2-PE biosynthesis are not clearly identified. Arabidopsis is a promising model system which is easily manipulated genetically to introduce new genes or pathways (Abdel-Ghany et al. 2013). In the present work, we introduced two 2-PE pathways from yeast and plants, which successfully produced 2-PE in transgenic Arabidopsis plants (Fig. 6). This highly efficient system can be employed to identify the putative genes isolated from other plant species highly accumulating 2-PE and its derivatives.

Many plant species can be utilized as the bioreactors for production of exogenetic proteins, polymers and commercially important chemicals by incorporation of synthetic pathways from microbes and other organisms (Börnke and Broer 2010; Gleba et al. 2007; Abdel-Ghany et al. 2013). This strategy has been considered as an economical and environmentally friendly alternative to chemical synthesis. In our study, we found that introducing 2-PE pathways not only led to the 2-PE accumulation, but also resulted in reduction of lignin content (Figs. 3, 6). However, the carbon flux towards 2-PE pathway was low compared with the lignin pathway. The biosynthesis of lignin was still dominant in transgenic Arabidopsis plants since the reconstructed 2-PE pathway is short and its storage pool is not large enough in plant cells. Therefore, it is not sufficient to redirect the substantial metabolic flux away from the lignin pathway by simply introducing 2-PE pathway to plants. To further generate the plants with low lignin, high saccharification, and high value-added chemicals, more valuable genes encoding enzymes which are capable of efficiently 
A
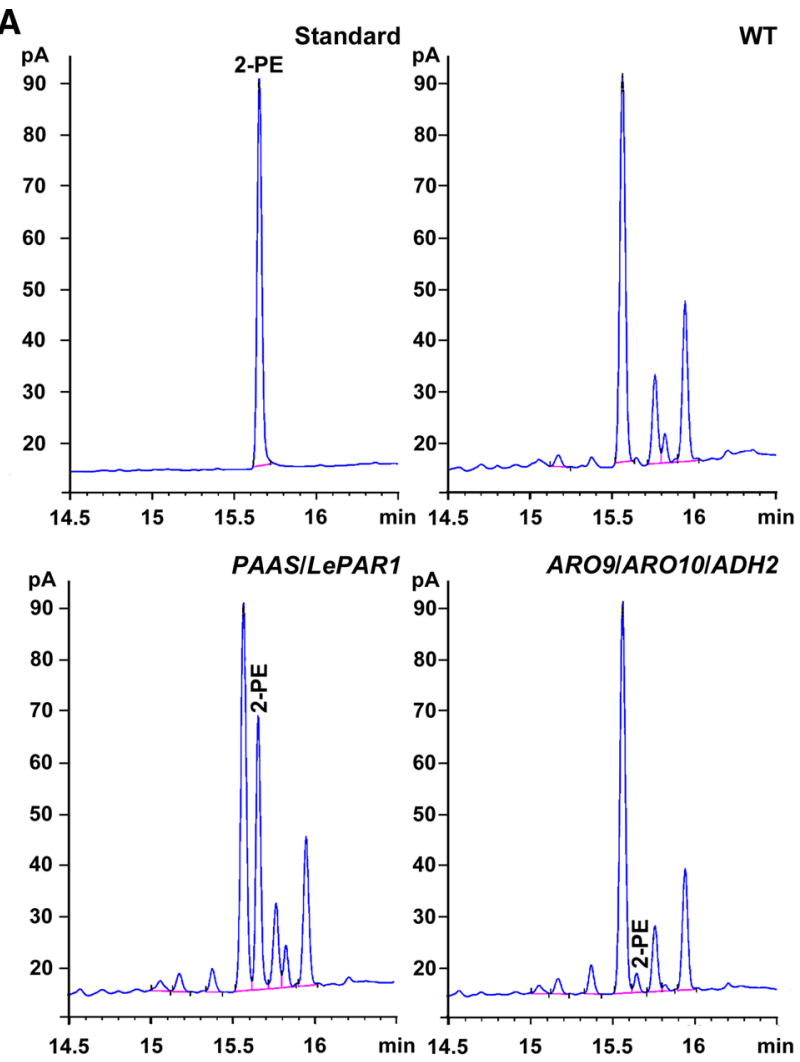

B

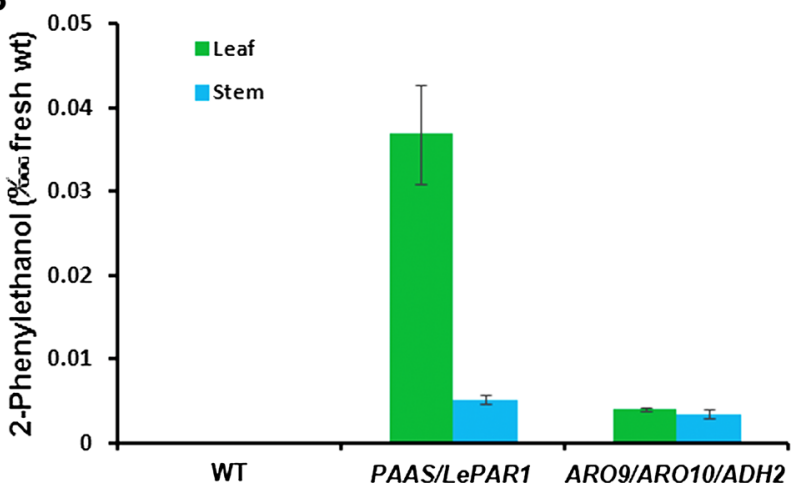

Fig. 6 2-Phenylethanol production in transgenic and wild-type plants. a GC analysis of 2-phenylethanol (2-PE) synthesis in leaves of 7-week-old wild-type, PAAS/LePAR1, and ARO9/ARO1O/ADH2 plants. b 2-Phenylethanol content in leaves and stems of 7-week-old PAAS/LePAR1, ARO9/ARO10/ADH2, and wild-type plants. Values are mean $\pm \mathrm{SE}$ of five independent biological repeats

converting the lignin precursors are worthy of recruitment and introducing into plant species with high biomass.

\section{Conclusion}

In summary, the attempt to reduce lignocellulosic biomass recalcitrance was achieved by introducing the reconstructed 2-PE biosynthetic pathways into Arabidopsis, which substantially disturbed lignin biosynthesis and therefore improved saccharification yield and produced value-added chemicals without adversely affecting plant growth and development. Our findings demonstrate that the carbon flux towards the biosynthesis of lignin could be efficiently reduced by engineering the competitive 2-PE biosynthetic pathway in plants. This proof-of-concept study thereby offers an alternative strategy to manipulate lignin for production of low cost biofuel and high valueadded commodity chemicals in biofuel crops, including poplar, corn, switchgrass, and miscanthus.

Author contribution statement G. Qi and D. Wang performed the gene cloning and plasmids construction, plant transformation and molecular identification of the transgenic plants, cell wall composition investigation, measurement of 2-phenylethanol, and drafted the manuscript cooperatively. L. Yu, X. Tang, G. Chai, G. He, W. $\mathrm{Ma}, \mathrm{S}$. Li, and Y. Kong participated in the cell wall composition investigation, helped in measurement of 2-phenylethanol and data analysis. C. Fu and G. Zhou conceived the project, supervised the analysis and critically revised the manuscript. All authors read and approved the final manuscript.

Acknowledgments This study was supported by the National Science and Technology Support Program (2013BAD22B01), the National Basic Research Program of China (2012CB114501), the National Natural Science Foundation of China (No. 31300502 and No. 31470291), China Postdoctoral Science Foundation, and the Youth Talent Plan of Chinese Academy of Agricultural Sciences to Y.Z.K.

Conflict of interest We declare that we have no conflict of interest.

\section{References}

Abdel-Ghany SE, Day I, Heuberger AL, Broeckling CD, Reddy AS (2013) Metabolic engineering of Arabidopsis for butanetriol production using bacterial genes. Metab Eng 20:109-120

Boerjan W, Ralph J, Baucher M (2003) Lignin biosynthesis. Annu Rev Plant Biol 54:519-546

Bonawitz ND, Chapple C (2013) Can genetic engineering of lignin biosynthesis be accomplished without an unacceptable yield penalty? Curr Opin Biotechnol 24:336-343

Bonawitz ND, Kim JI, Tobimatsu Y, Ciesielski PN, Anderson NA, Ximenes E, Maeda J, Ralph J, Donohoe BS, Ladisch M, Chapple C (2014) Disruption of Mediator rescues the stunted growth of a lignin-deficient Arabidopsis mutant. Nature 509(7500):376-380

Börnke F, Broer I (2010) Tailoring plant metabolism for the production of novel polymers and platform chemicals. Curr Opin Plant Biol 13(3):354-362

Campbell MM, Sederoff RR (1996) Variation in lignin content and composition. Mechanisms of control and implications for the genetic improvement of plants. Plant Physiol 110:3-13

Chai G, Wang Z, Tang X, Yu L, Qi G, Wang D, Yan X, Kong Y, Zhou G (2014) R2R3-MYB gene pairs in Populus: evolution and contribution to secondary wall formation and flowering time. J Exp Bot 65(15):4255-4269 
Chen F, Dixon RA (2007) Lignin modification improves fermentable sugar yields for biofuel production. Nat Biotechnol 25:759-761

Douglas CJ (1996) Phenylpropanoid metabolism and lignin biosynthesis: from weeds to trees. Trends Plant Sci 1:171-178

Eudes A, George A, Mukerjee P, Kim JS, Pollet B, Benke PI, Yang F, Mitra P, Sun L, Cetinkol OP, Chabout S, Mouille G, SoubigouTaconnat L, Balzergue S, Singh S, Holmes BM, Mukhopadhyay A, Keasling JD, Simmons BA, Lapierre C, Ralph J, Loqué D (2012) Biosynthesis and incorporation of side-chain-truncated lignin monomers to reduce lignin polymerization and enhance saccharification. Plant Biotechnol J 10(5):609-620

Eudes A, Liang Y, Mitra P, Loqué D (2014) Lignin bioengineering. Curr Opin Biotechnol 26:189-198

Foster CE, Martin TM, Pauly M (2010) Comprehensive compositional analysis of plant cell walls (lignocellulosic biomass) part II: carbohydrates. J Vis Exp (37)

Fu C, Mielenz JR, Xiao X, Ge Y, Hamilton CY, Rodriguez M Jr, Chen F, Foston M, Ragauskas A, Bouton J, Dixon RA, Wang ZY (2011a) Genetic manipulation of lignin reduces recalcitrance and improves ethanol production from switchgrass. Proc Natl Acad Sci USA 108(9):3803-3808

Fu C, Xiao X, Xi Y, Ge Y, Chen F, Bouton J, Dixon RA, Wang ZY (2011b) Downregulation of cinnamyl alcohol dehydrogenase (CAD) leads to improved saccharification efficiency in switchgrass. Bioenerg Res 4(3):153-164

Gleba Y, Klimyuk V, Marillonnet S (2007) Viral vectors for the expression of proteins in plants. Curr Opin Biotechnol 18:134-141

Guo D, Chen F, Inoue K, Blount JW, Dixon RA (2001a) Downregulation of caffeic acid 3-O-methyltransferase and caffeoyl CoA 3-O-methyltransferase in transgenic alfalfa. impacts on lignin structure and implications for the biosynthesis of $\mathrm{G}$ and $\mathrm{S}$ lignin. Plant Cell 13:73-88

Guo D, Chen F, Wheeler J, Winder J, Selman S, Peterson M, Dixon RA (2001b) Improvement of in-rumen digestibility of alfalfa forage by genetic manipulation of lignin $O$-methyltransferases. Transgenic Res 10:457-464

Hazelwood LA, Daran JM, van Maris AJ, Pronk JT, Dickinson JR (2008) The Ehrlich pathway for fusel alcohol production: a century of research on Saccharomyces cerevisiae metabolism. Appl Environ Microbiol 74(8):2259-2266

Hu WJ, Harding SA, Lung J, Popko JL, Ralph J, Stokke DD, Tsai CJ, Chiang VL (1999) Repression of lignin biosynthesis promotes cellulose accumulation and growth in transgenic trees. Nat Biotechnol 17(8):808-812

Jouanin L, Goujon T, de Nadaï V, Martin MT, Mila I, Vallet C, Pollet B, Yoshinaga A, Chabbert B, Petit-Conil M, Lapierre C (2000) Lignification in transgenic poplars with extremely reduced caffeic acid $O$-methyltransferase activity. Plant Physiol 123:1363-1374

Kaminaga Y, Schnepp J, Peel G, Kish CM, Ben-Nissan G, Weiss D, Orlova I, Lavie O, Rhodes D, Wood K, Porterfield DM, Cooper AJ, Schloss JV, Pichersky E, Vainstein A, Dudareva N (2006) Plant phenylacetaldehyde synthase is a bifunctional homotetrameric enzyme that catalyzes phenylalanine decarboxylation and oxidation. J Biol Chem 281(33):23357-23366

Karimi M, Bleys A, Vanderhaeghen R, Hilson P (2007) Building blocks for plant gene assembly. Plant Physiol 145(4):1183-1191

Li X, Weng JK, Chapple C (2008) Improvement of biomass through lignin modification. Plant J 54(4):569-581

Li M, Xiong G, Li R, Cui J, Tang D, Zhang B, Pauly M, Cheng Z, Zhou Y (2009) Rice cellulose synthase-like D4 is essential for normal cell-wall biosynthesis and plant growth. Plant $\mathbf{J}$ 60(6):1055-1069

Li X, Ximenes E, Kim Y, Slininger M, Meilan R, Ladisch M, Chapple C (2010) Lignin monomer composition affects Arabidopsis cell- wall degradability after liquid hot water pretreatment. Biotechnol Biofuels 3:27

Louie GV, Bowman ME, Tu Y, Mouradov A, Spangenberg G, Noel JP (2010) Structure-function analyses of a caffeic acid $O$ methyltransferase from perennial ryegrass reveal the molecular basis for substrate preference. Plant Cell 22:4114-4127

Pomar F, Merino F, Barceló AR (2002) O-4-Linked coniferyl and sinapyl aldehydes in lignifying cell walls are the main targets of the Wiesner (phloroglucinol- $\mathrm{HCl}$ ) reaction. Protoplasma 220(1-2):17-28

Qi G, Hu R, Yu L, Chai G, Cao Y, Zuo R, Kong Y, Zhou G (2013) Two poplar cellulose synthase-like D genes, PdCSLD5 and PdCSLD6, are functionally conserved with Arabidopsis CSLD3. J Plant Physiol 170(14):1267-1276

Ragauskas AJ, Beckham GT, Biddy MJ, Chandra R, Chen F, Davis MF, Davison BH, Dixon RA, Gilna P, Keller M, Langan P, Naskar AK, Saddler JN, Tschaplinski TJ, Tuskan GA, Wyman CE (2014) Lignin valorization: improving lignin processing in the biorefinery. Science 344(6185):1246843

Reddy MS, Chen F, Shadle G, Jackson L, Aljoe H, Dixon RA (2005) Targeted down-regulation of cytochrome P450 enzymes for forage quality improvement in alfalfa (Medicago sativa L.). Proc Natl Acad Sci USA 102:16573-16578

Rusanov K, Kovacheva N, Vosman B, Zhang L, Rajapakse S, Atanassov A, Atanassov I (2005) Microsatellite analysis of Rosa damascena Mill. accessions reveals genetic similarity between genotypes used for rose oil production and old Damask rose varieties. Theor Appl Genet 111(4):804-809

Sakai M, Hirata H, Sayama H, Sekiguchi K, Itano H, Asai T, Dohra H, Hara M, Watanabe N (2007) Production of 2-phenylethanol in roses as the dominant floral scent compound from L-phenylalanine by two key enzymes, a PLP-dependent decarboxylase and a phenylacetaldehyde reductase. Biosci Biotechnol Biochem 71(10):2408-2419

Samuel R, Pu Y, Jiang N, Fu C, Wang ZY, Ragauskas A (2014) Structural characterization of lignin in wild-type versus COMT down-regulated switchgrass. Front Energy Res 1:1-9

Sarath G, Mitchell RB, Sattler SE, Funnell D, Pedersen JF, Graybosch RA, Vogel KP (2008) Opportunities and roadblocks in utilizing forages and small grains for liquid fuels. J Ind Microbiol Biotechnol 35:343-354

Sewalt VJH, Ni W, Blount JW, Jung HG, Masoud SA, Howles PA, Lamb C, Dixon RA (1997a) Reduced lignin content and altered lignin composition in transgenic tobacco down-regulated in expression of L-phenylalanine ammonia-lyase or cinnamate 4-hydroxylase. Plant Physiol 115:41-50

Sewalt VJH, Ni WT, Jung HG, Dixon RA (1997b) Lignin impact on fiber degradation: increased enzymatic digestibility of genetically engineered tobacco (Nicotiana tabacum) stems reduced in lignin content. J Agric Food Chem 45:1977-1983

Shen B, Li C, Tarczynski MC (2002) High free-methionine and decreased lignin content result from a mutation in the Arabidopsis S-adenosyl-L-methionine synthetase 3 gene. Plant J 29(3):371-380

Shen H, He X, Poovaiah CR, Wuddineh WA, Ma J, Mann DG, Wang H, Jackson L, Tang Y, Stewart CN Jr, Chen F, Dixon RA (2012) Functional characterization of the switchgrass (Panicum virgatum) R2R3-MYB transcription factor PvMYB4 for improvement of lignocellulosic feedstocks. New Phytol 193(1):121-136

Tang HM, Liu S, Hill-Skinner S, Wu W, Reed D, Yeh CT, Nettleton D, Schnable PS (2014) The maize brown midrib2 (bm2) gene encodes a methylenetetrahydrofolate reductase that contributes to lignin accumulation. Plant J 77(3):380-392

Tieman DM, Loucas HM, Kim JY, Clark DG, Klee HJ (2007) Tomato phenylacetaldehyde reductases catalyze the last step in the synthesis of the aroma volatile 2-phenylethanol. Phytochemistry 68(21):2660-2669 
Tu Y, Rochfort S, Liu Z, Ran Y, Griffith M, Badenhorst P, Louie GV, Bowman ME, Smith KF, Noel JP, Mouradov A, Spangenberg G (2010) Functional analyses of caffeic acid $O$-methyltransferase and cinnamoyl-CoA-reductase genes from perennial ryegrass (Lolium perenne). Plant Cell 22:3357-3373

Van Acker R, Vanholme R, Storme V, Mortimer JC, Dupree P, Boerjan W (2013) Lignin biosynthesis perturbations affect secondary cell wall composition and saccharification yield in Arabidopsis thaliana. Biotechnol Biofuels 6(1):46

Vanholme R, Demedts B, Morreel K, Ralph J, Boerjan W (2010) Lignin biosynthesis and structure. Plant Physiol 153(3):895-905

Vogt T (2010) Phenylpropanoid biosynthesis. Mol Plant 3(1):2-20

Ximenes E, Kim Y, Mosier N, Dien B, Ladisch M (2010) Inhibition of cellulases by phenols. Enzyme Microb Tech 46:170-176
Yu L, Zhang X, Li SS, Liu XY, Sun L, Liu HB, Iteku J, Zhou YF, Tai GH (2010) Rhamnogalacturonan I domains from ginseng pectin. Carbohydr Polym 79(4):811-817

Zhang K, Bhuiya MW, Pazo JR, Miao Y, Kim H, Ralph J, Liu CJ (2012) An engineered monolignol 4-o-methyltransferase depresses lignin biosynthesis and confers novel metabolic capability in Arabidopsis. Plant Cell 24(7):3135-3152

Zhao Q, Tobimatsu Y, Zhou R, Pattathil S, Gallego-Giraldo L, Fu C, Jackson LA, Hahn MG, Kim H, Chen F, Ralph J, Dixon RA (2013) Loss of function of cinnamyl alcohol dehydrogenase 1 leads to unconventional lignin and a temperature-sensitive growth defect in Medicago truncatula. Proc Natl Acad Sci USA 110(33):13660-13665 